\title{
A NEW POSSIBLE RESONANCE FOR POPULATION II CEPHEIDS
}

\author{
A. N. $\operatorname{cox}$ \\ Theoretical Division, Los Alamos National Laboratory, \\ University of California
}

R. B. KIDMAN

Theoretical Division, Los Alamos National Laboratory, University of California

Light and velocity curves of some radial mode variable stars seem to indicate a resonance where the second overtone has a period exactly half that of the fundamental mode. The two classes of stars that show this resonance by bumps in their light curves are the classical Cepheids and the population II BL Her variables. We here propose that there is another resonance for the population II $W$ Vir variables where the ratio of the first overtone to the fundamental periods is 0.5 .

Observations by Kwee (1967) show that these population II Cepheids, have frequently a crested or a flattop shape of their light curve. Some light curves seem to be featureless, but that may be only the result of too few observations resulting in too few phase points.

Crested light curves such as for W Vir show a bump or standstill on descending light rather like the classical Cepheids with periods between 5 and 10 days. The flattop type of light curves sometimes have bumps on rising light as for Cepheids with periods between 10 and 20 days. The problem with these variable stars is that they do not have a correlation of these light curve shapes with period as long-ago demonstrated by Hertzsprung (1926) for the classical Cepheids. The two types of light curves both exist all the way from about 10 to 20 day periods.

BL Her variables that show bumps in their light and velocity curves have some correlation of the light curve shape with period, but it is not a perfect one. King, Cox and Hodson (1981) have shown that on the Hertzsprung-Russell diagram, lines of constant period (slightly steeper than lines of constant radius) have smaller slopes than the slightly curved lines of constant period ratio $\mathrm{P}_{2} / \mathrm{P}_{0}$. This has also been shown again by Hodson, Cox and King (1982) using the Carson opacities. In this latter case, the period ratios are a bit smaller, but that is a direct result of the larger Carson opacities that deconcentrate the outer envelope structure in a way that $P_{0}$ is increased more than $P_{2}$. As is well known, this smaller period ratio for the BL Her variables does not affect the bump mass determination in any significant way, but the smaller period ratio using the Carson opacities for the classical Cepheids can compensate for otherwise smaller bump masses (Vemury and Stothers, 1978).

The easy visibility of the Hertzsprung sequence for the classical Cepheids is because the lines in the Hertzsprung-Russell diagram of constant period and those of constant period ratio $P_{2} / P_{0}$ are parallel, 
resulting in an almost perfect correlation between $P_{0}$ and $P_{2} / P_{0}$. Both red and blue Cepheids with a given $\mathrm{P}_{0}$ show a bump at the same phase.

The difference in slope between the lines of constant period and constant period ratio $P_{2} / P_{0}$ for the $B L$ Her variables means that at a given period, a range of period ratios is possible. Then the phase of the bumps is not fixed, but it is confined to a small range. This explains why BL Her itself has a bump on falling light with a period ratio inferred to be 0.52 or 0.53 , whereas $X X$ Vir at the same 1.3 day period has no bump, implying a period ratio of greater than 0.53 which can occur if XX Vir is bluer, fainter, or more massive than BL Her.

Simon and Schmidt (1976) showed that this correlation of bump phase with period can be seen in the results of the nonlinear hydrodynamic integrations that have been done by Stobie (1969) and many others. A unique relation was found that made it possible to predict the bump phase as seen in the theoretical light and velocity curves by knowing the period ratio $P_{2} / P_{0}$, if this ratio was between 0.46 and 0.53 . From the recent work of Buchler (1984), we see that the bump is actually the result of a resonance between these two modes.

Standard models with masses and luminosities that are consistent with evolution theory give linear theory period ratios too large for a given period. For example, for a $7 \mathrm{M}_{\theta}$ Cepheid and the evolution theory luminosity of just over 3700 solar luminosities, on the blue side of the instability strip this star would have a period of 6 days while on the red side of the strip its period would be 12 days. In these cases the predicted period ratio would range from about 0.57 to 0.53 , but the appearance of observed light curve bumps indicate period ratios ranging from about 0.52 to 0.49 . The original way to reconcile this discrepancy was to lower the mass to about $4 M_{0}^{\prime} s$ at this luminosity. Then the period ratios from linear theory and the bumps seen in nonlinear hydrodynamic integration light and velocity curves fit the data. Actually this has recently been considered more of a period ratio anomaly than a mass anomaly, and ways to deconcentrate the Cepheid envelope and reduce the period ratio have been sought.

Of the three currently viable ways of deconcentrating the outer 0.01 to 0.1 per cent of the stellar mass, perhaps only one is now possible. The large magnetic fields postulated by Stothers (1979) can strongly affect the stellar structure, but their existence to give a pressure as large as the gas pressure has not been (and maybe cannot be) measured. If the required fields are generated by a convective dynamo, they are many powers of ten too large. A primordial field may be strong enough, but then all the Cepheids in this mass range of 5 to $9 \mathrm{M}_{\theta}$ need to have had a rather uniform early formation history.

The excellent suggestion by Simon (1982) that the envelope can be deconcentrated with a higher metal opacity must be rejected because such an effect does not seem possible from the viewpoint of atomic physics and the compositions that much exist in Cepheid envelopes. Actually some of this very effect has been seen earlier because the higher Carson 
opacities at temperature above $5 \times 10^{5} \mathrm{~K}$ did slightly deconcentrate the stellar models, especially for the population I case where there is a significant CNONe abundance. The discovery that the Carson opacities are inaccurate in this high temperature region by Carson, Huebner, Magee, and Merts (1984) has removed the earlier mass anomaly improvements, and the parallel result by Magee, Merts, and Huebner (1984) that no other opacity source is available, seem to eliminate the enhanced opacity explanation for the period ratio anomaly.

We therefore want to reemphasize that an enhanced helium abundance in the Cepheid envelope can deconcentrate the density structure and reconcile both the bump and beat Cepheid mass anomalies. Cox, Michaud, and Hodson (1978) have shown that a Cepheid wind, if it is anything like the solar wind, can blow away more hydrogen than helium, leaving a very thin homogeneous convective zone that is enhanced to a mass fraction as large as 0.65 or even possibly 0.75 .

This helium enhancement takes time. A $7 \mathrm{M}_{\theta}$ star evolving on its blue loops from the red giant region with a very deep convective envelope has only 1-10 million years before it becomes a second crossing Cepheid. At $8 \mathrm{M}_{\theta}$ this time is reduced so much that very little wind enhancement is possible. Therefore the very blue $8 \mathrm{M}$ Cepheid with a period of 10 or more days should not be deconcentrated, and it should not show any period ratio anomaly for periods up to 20 days as it evolves back to the red edge of the instability strip.

Recent observations and interpretations by Davis, Moffett, and Barnes (1981) show that $X \mathrm{Cyg}$ at 16.4 days shows little or no period ratio (bump phase) anomaly. Thus enhanced helium is not needed or predicted for the masses above about $8 \mathrm{M}$. Unlike the tangled magnetic field or the increased opacity proposals, which do not have a reversion to standard models at greater than $8 \mathrm{M}_{\theta}$ or a period of about 15 days, the enhanced helium model slowly merges with non-enhanced models at this mass and period. Bumps on rising light are seen for periods as long as 26 days for the Cepheid X Pup as observed by Pel (1976).

Returning to the population II Cepheids, we see in Figure 1 that lines of constant $P_{1} / P_{0}$ near 0.5 are in the very region where the crested and flattop type light curves occur. In this figure we show only globular cluster variables where their luminosities are rather well determined. The symbols $b$ and $B$ stand for, respectively, BL Her variables in $w$ Cen and in all the other globular clusters. The $f$ and $F$ symbols refer to again, respectively, the $w$ Cen and other globular cluster variables with flattop light curves. The $c$ and $C$ symbols refer to the crested light curves and the $r$ and $R$ symbols refer to two RV Tau variables.

Constant period lines for the BL Her variables have been computed for a mass of $0.65 \mathrm{M}_{\theta}$ with the King Ia table with a $Z$ composition of 0.001 . The Stellingwerf (1975ab) fit for the opacity and equation of state has been used for the $0.60 \mathrm{M}_{\theta} \mathrm{W}$ Vir lines at higher luminosities. The location of the blue edge and the curvature of the constant period lines may be changed when the actual tabular data are used. It has been 
verified that both the fit and the tablular material properties give constant $P_{1} / P_{0}$ lines that go right up the instability strip at least approximately as drawn. Thus the flattop curves, with often bumps on rising light and maybe a period ratio of less than 0.5 should be always redder than the crested light curve cases as observed.

Figure 1. On the Hertzsprung-Russell diagram lines of constant period and lines of constant period ratio $P_{1} / P_{0}$ are drawn for $0.65 \mathrm{M}_{\theta}$ for the $B L$ Her luminosities and for $0.60 \mathrm{M}_{\theta}$ for the $W \mathrm{Vir}$ variables. Individual stars in globular clusters and their periods are also plotted.

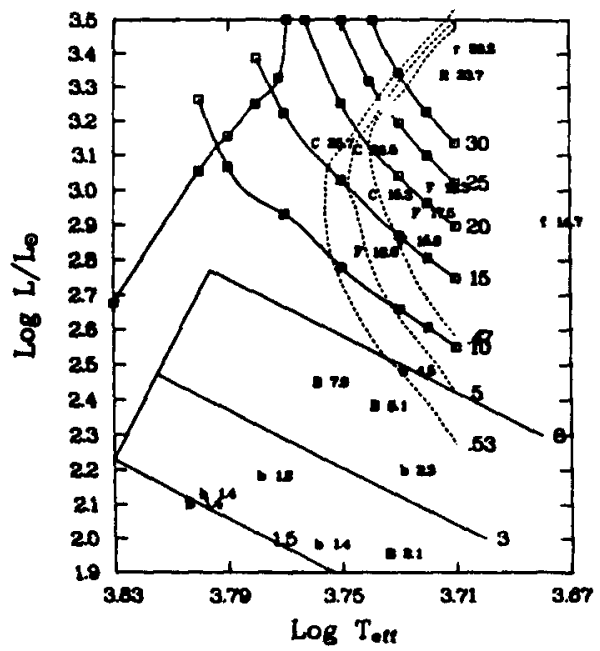

\section{REFERENCES}

Buchler, J.R. (1984). Ap. J., in press.

Carson, T.R., Huebner, W.F., Magee, N.H., \& Merts, A.L. (1984). Ap. J., (Aug 15).

Cox, A.N., Michaud, G., \& Hodson, S.W. (1978). Ap. J., 222, 621.

Davis, C.G., Moffett, T.J., \& Barnes, T.G. (1981). Ap. J., 246, 914.

Hertzsprung, E. (1926). Bull. Astr. Int. Netherlands, 3,115 .

Hodson, S.W., Cox, A.N., \& King, D.S. (1982). Ap. J., 253, 260.

King, D.S., Cox, A.N., \& Hodson, S.W. (1981). Ap. J., $\frac{244}{6}, 242$.

Kwee, K.K. (1967). Bull. Astr. Int. Netherlands, $19,260$.

Magee, N.H., Merts, A.L. \& Huebner, W.F. (1984). Ap. J., (Aug 15).

Pe1, J.W. (1976). Astr. Ap. Supp1., 24, 413.

Simon, N.R. \& Schmidt, E.G. (1976). Ap. J., 205, 162.

Simon, N.R. (1982). Ap. J., Lett 260, L87.

Stellingwerf, R.F. (1975a). Ap. J., 195, 441.

Stellingwerf, R.F. (1975b). Ap. J., 199,705 .

Stobie, R.S. (1969). M.N.R.A.S., 144, 485 .

Stothers, R. (1979). Ap. J., 234, 257 .

Vemury, S.K. \& Stothers, R. (1978). Ap. J., 225, 939. 